\title{
Combination probes for stagnation pressure and temperature measurements in gas turbine engines
}

\author{
C Bonham ${ }^{1}$, S J Thorpe ${ }^{1} \ddagger$, M N Erlund ${ }^{2}$, R J Stevenson ${ }^{2}$ \\ ${ }^{1}$ Department of Aeronautical and Automotive Engineering, Loughborough \\ University, Loughborough, LE11 3TU, UK \\ 2 Rolls-Royce plc, PO Box 31, Derby, DE24 8BJ, UK \\ E-mail: c.bonham@lboro.ac.uk
}

\begin{abstract}
During gas turbine engine testing, steady-state gas-path stagnation pressures and temperatures are measured in order to calculate the efficiencies of the main components of turbomachinery. These measurements are acquired using fixed intrusive probes, which are installed at the inlet and outlet of each component at discrete point locations across the gas-path. The overall uncertainty in calculated component efficiency is sensitive to the accuracy of discrete point pressures and temperatures, as well as the spatial sampling across the gas-path. Both of these aspects of the measurement system must be considered if more accurate component efficiencies are to be determined. High accuracy has become increasingly important as engine manufacturers have begun to pursue small gains in component performance, which require efficiencies to be resolved to within less than $\pm 1 \%$. This article reports on three new probe designs that have been developed in a response to this demand. The probes adopt a compact combination arrangement that facilitates up to twice the spatial coverage compared to individual stagnation pressure and temperature probes. The probes also utilise novel temperature sensors and high recovery factor shield designs that facilitate improvements in point measurement accuracy compared to standard Kiel probes used in engine testing. These changes allow efficiencies to be resolved within $\pm 1 \%$ over a wider range of conditions than is currently achievable with Kiel probes.
\end{abstract}

Keywords Combination probes, stagnation pressure, stagnation temperature, recovery factor, spatial resolution

Submitted to: Meas. Sci. Technol. 
Combination probes for stagnation pressure and temperature measurements in gas turbine engines2

\section{Introduction}

During the first half of the 20th century, the rapid growth of aviation led to renewed interest in the measurement of fluid properties in high-velocity flows. This resulted in the development of fixed intrusive probes, capable of detecting stagnation pressures and temperatures by bringing the fluid to rest adiabatically.

In 1935, Kiel described a stagnation pressure probe designed for use in the determination of aircraft flight velocity (Kiel 1935). This probe featured a venturishaped cylindrical shield that housed a pitot tube connected to a remote pressure transducer. The cylinder was provided to reduce the probe's sensitivity to pitch and yaw angles by deflecting the oncoming flow towards the axis of the pitot tube. Markowski and Moffatt (1948) reported that this arrangement could yield accurate stagnation pressure measurements at flow incidence angles up to $\pm 55^{\circ}$.

In 1940, Franz described a stagnation temperature probe intended for use in aircraft engine superchargers (Franz 1940). The device adopted a similar design to the Kiel probe, but replaced the pitot tube with a thermocouple sensor. This arrangement was intended to facilitate the measurement of stagnation temperature by adiabatically decelerating the flow travelling over the sensor to a low velocity. However, it was established that the measured stagnation temperature was also sensitive to external heat transfer effects (Moffat 1962). This led to the development of more complex probes, designed to reduce the effects of unwanted conductive and radiative heat transfer between the sensor and the surroundings. Examples of such probes are described by Mullikin (1941), King (1943) and Stanworth (1962).

Today, probes similar to those described by Kiel and Franz are used to measure steady-state gas-path stagnation pressures and temperatures during gas turbine engine testing. These measurements are primarily used to calculate turbomachinery component efficiencies, which are vital for determining whether the engine is operating as intended or whether design refinements are required. These efficiencies are typically determined from area-weighted averages of discrete point measurements, acquired by stagnation pressure and temperature probes located at the inlet and outlet of each component (Cumpsty \& Horlock 2006). At each axial measurement plane, probes are installed radially across the gas-path at several circumferential locations. This is intended to allow spatial variations in stagnation pressure and temperature to be resolved and representative gas-path averages to be calculated.

The number of probes installed at any axial location within the engine is restricted by concerns over parasitic pressure losses. On an instrumented development engine, these losses can lead to significant changes in component performance relative to a production machine. Lepicovsky (2008) and Ng \& Coull (2017) have conducted investigations into the pressure losses caused by probes installed in compressor and turbine rigs. In this work, the loss attributed to turbine leading edge instrumentation was shown to scale linearly with the total probe frontal area. To accurately characterise component performance, the proportion of the gas-path obstructed by pressure and 
temperature probes must therefore be constrained. Saravanamutto (1990) recommends that this obstruction be limited to $2-5 \%$ of the available flow area.

Balancing the conflicting requirements of high sampling resolution and low flow path blockage is most challenging when annulus dimensions are small. In principle, non-intrusive techniques offer the best means of achieving high spatial coverage whilst maintaining low flow path blockage. Although such techniques have been trialled in gas turbine engine testing (see for example Otero et al (2016)), they are still in the development phase and not routinely employed for gas-path measurements. As an alternative, Allan (1983) proposed the use of traversing probes to spatially sample the gas-path stagnation pressure and temperature. However, this approach incurs additional cost and complexity relative to fixed probes, and results in varying flow path blockage over the traverse cycle. With fixed probes, the greatest coverage can be achieved with combination probes, in which the stagnation pressure and temperature are measured at a common location within a single probe head. This arrangement is more compact compared to separate stagnation pressure and temperature probes, and therefore permits a greater number of probes to be employed for a given flow path blockage.

The current article reports on three new combination probes that have been specifically developed to achieve improved stagnation pressure and temperature accuracy compared to the current state-of-the-art. Although combination probes have previously been described by Glawe et al (1968) and Krause et al (1972), the new probes seek to improve on these designs by adopting: 1) platinum resistance thermometers for higher fundamental temperature sensing accuracy, as well as 2) high pressure and temperature recovery factor shields that ensure low levels of measurement correction are required. Together, the combination of more accurate point measurements and increased spatial measurement resolution offered by the new probes is intended to deliver the reductions in uncertainty needed to resolve turbomachinery component efficiencies to within less than $\pm 1 \%$. In practice, this requires stagnation pressure and temperature uncertainties of $\pm 0.1 \%$ to be achieved.

\section{Accuracy considerations}

Equation 1 shows how the isentropic efficiency of a compressor can be calculated from the gas-path stagnation pressure and temperature at the inlet and outlet of the component:

$$
\eta=\frac{\left[\frac{P_{o, \text { out }}}{P_{o, \text { in }}}\right]^{\frac{\gamma-1}{\gamma}}-1}{\left[\frac{T_{o, \text { out }}}{T_{o, \text { in }}}\right]-1}
$$

In this equation, the stagnation pressures and temperatures represent area-weighted averages that are determined using point measurements acquired from discrete probes positioned across the gas-path. The accurate assessment of turbomachinery efficiency has become increasingly important as engine manufacturers have begun to pursue small improvements in component performance. This has been driven by the success of 
past engine development programs, in which meticulous rig testing and sophisticated computational modelling have delivered significant performance gains. To facilitate the assessment of turbomachinery efficiency to within less than $\pm 1 \%$, overall uncertainties of $\pm 0.1 \%$ in average stagnation pressures and temperatures must be sought. These uncertainties are sensitive to the accuracy of the discrete point measurements, as well as the spatial sampling across the gas-path.

\subsection{Measurement accuracy}

The accuracy of point stagnation pressure and temperature measurements is determined by uncertainties introduced by the sensor and data acquisition system, as well as the recovery performance of the probe. The impact of these phenomena on the calculated efficiency is most pronounced for low pressure ratio components, since any uncertainty constitutes a greater proportion of the change in stagnation pressure or temperature across the component (Saravanamuttoo 1990). This is illustrated in Figure 1, which shows the impact of different uncertainties in stagnation pressure and temperature on the isentropic efficiency of a compressor operating over a range of pressure ratios.

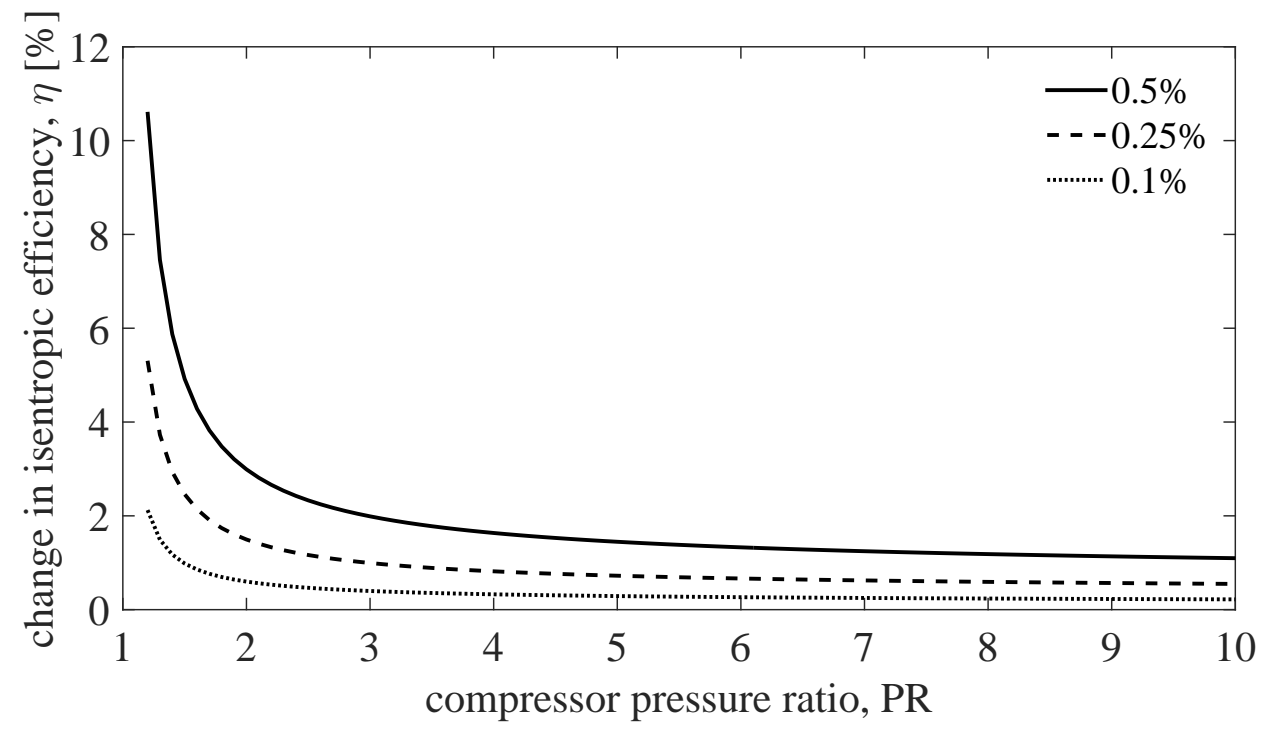

Figure 1. A graph showing the impact of uncertainties in stagnation pressure and temperature on the calculated isentropic efficiency of a compressor $\left(T_{o, \text { in }}=300 \mathrm{~K}, \eta_{p}=\right.$ $0.85)$.

With $\pm 0.1 \%$ uncertainties in stagnation pressure and temperature, Figure 1 shows that the compressor isentropic efficiency can be assessed to an accuracy of $< \pm 1 \%$ for all pressure ratios $\geq 1.5$. This corresponds to the lowest pressure ratio that would be expected across the fan of a high-bypass ratio turbofan engine. However, such low levels of measurement uncertainty cannot currently be achieved in an engine test environment. With higher uncertainties, it is clear from Figure 1 that an accuracy of $< \pm 1 \%$ in compressor isentropic efficiency becomes increasingly difficult to achieve 
at low pressure ratios. As a consequence, particular importance is placed on reducing stagnation pressure and temperature uncertainties in components operating at these conditions.

In an engine environment, stagnation pressure and temperature uncertainties can be impacted by a number of factors. Signals from thermocouples and pressure transducers can become corrupted by static calibration drift and electrical noise contamination. In the authors' experience, these effects can introduce uncertainties of up to $\pm 0.27 \%$ for thermocouples at ambient temperature conditions due to the sensor's low thermoelectric response $\left(40 \mu \mathrm{V} /{ }^{\circ} \mathrm{C}\right.$ for $\mathrm{N}$-type devices). The effects have less impact for pressure transducers, where signal levels are higher and uncertainties of $\pm 0.05 \%$ can typically be achieved (Massini, Miller, Hodson \& Collings 2010). In the gas-path, the high-subsonic Mach numbers can also lead to under-reads in stagnation pressure and temperature due to the limitations of probe recovery performance. These under-reads tend to be greatest for stagnation temperature measurements, since they are sensitive to recovery effects of the flow over the thermocouple sensor, as well as heat transfer effects between the sensor and the surroundings. A detailed description of these effects is provided by Moffat (1962). Under-reads in stagnation pressure measurements are usually smaller, as they are predominately related to the anisentropic deceleration of the flow provided that no inlet separation occurs. Under-reads in stagnation pressure and temperature can be corrected post-test using probe recovery factors determined via aerodynamic calibration. However, these corrections still introduce additional sources of uncertainty beyond those associated with sensors and data acquisition systems.

\subsection{Spatial sampling}

The accuracy of area-weighted average stagnation pressures and temperatures is additionally affected by the sampling of the flow field. This is influenced by the number of discrete probe measurements used to average the entire gas-path at a particular axial location, as well as the local averaging that occurs across each probe's inlet area. Provided that probe dimensions are small relative to spatial flow variations, averaging across the probe inlets will be of secondary importance compared to averaging across the gas-path. The number of probe measurements needed to obtain representative gaspath averages is strongly related to the uniformity of the flow field. Comparatively few measurements are required at the engine inlet where the flow is relatively uniform, but more measurements are required at compressor exit where wakes from upstream blade rows distort the flow field (Saravanamuttoo 1990). These wakes also cause periodic fluctuations in gas-path stagnation pressure and temperature that influence the timeaverage measurements provided by the probes. A thorough analysis of these effects can be found in Agnew et al (1985).

Figure 2 indicates the number of radial measurements needed at a single circumferential location to resolve the isentropic efficiency of compressor operating at 3 different pressure ratios. In this figure, point measurement locations are distributed 
Combination probes for stagnation pressure and temperature measurements in gas turbine engines6

uniformly across the height of the gas-path and the flow is assumed to vary sinusoidally according to eq. 2 and eq. 3 ;

$$
\begin{aligned}
& T R=T R_{\text {wall }}\left[1+A \sin \left(\pi \frac{r-r_{i}}{r_{o}-r_{i}}\right)\right] \\
& P R=P R_{\text {wall }}\left[1+B \sin \left(\pi \frac{r-r_{i}}{r_{o}-r_{i}}\right)\right]
\end{aligned}
$$

where $A=0.1$ and $B=0.05$.

To determine the compressor efficiency to within $< \pm 0.1 \%$, Figure 2 shows that at least 7 measurements are needed in order to resolve radial flow variations at a single circumferential location. A similar analysis of circumferential flow variations at a single radial location reveals that at least 5 measurements are also needed around the perimeter of the gas-path. This corresponds to a total requirement of 35 measurements of both stagnation pressure and temperature. With individual stagnation pressure and temperature probes, a total of 70 probes must therefore be distributed over the gaspath. Such probe numbers may be difficult to accommodate in the engine, particularly where small annulus dimensions mean that the flow path blockage can become high. To maintain an acceptable level of blockage, probe numbers may consequently be restricted leading to higher uncertainties in efficiency. With combination probes, however, two measurements are acquired at each location, halving the number of probes required and the attendant flow path blockage. Comparatively more accurate assessments of compressor efficiency may therefore be performed, for little increase in instrumentation cost and complexity.

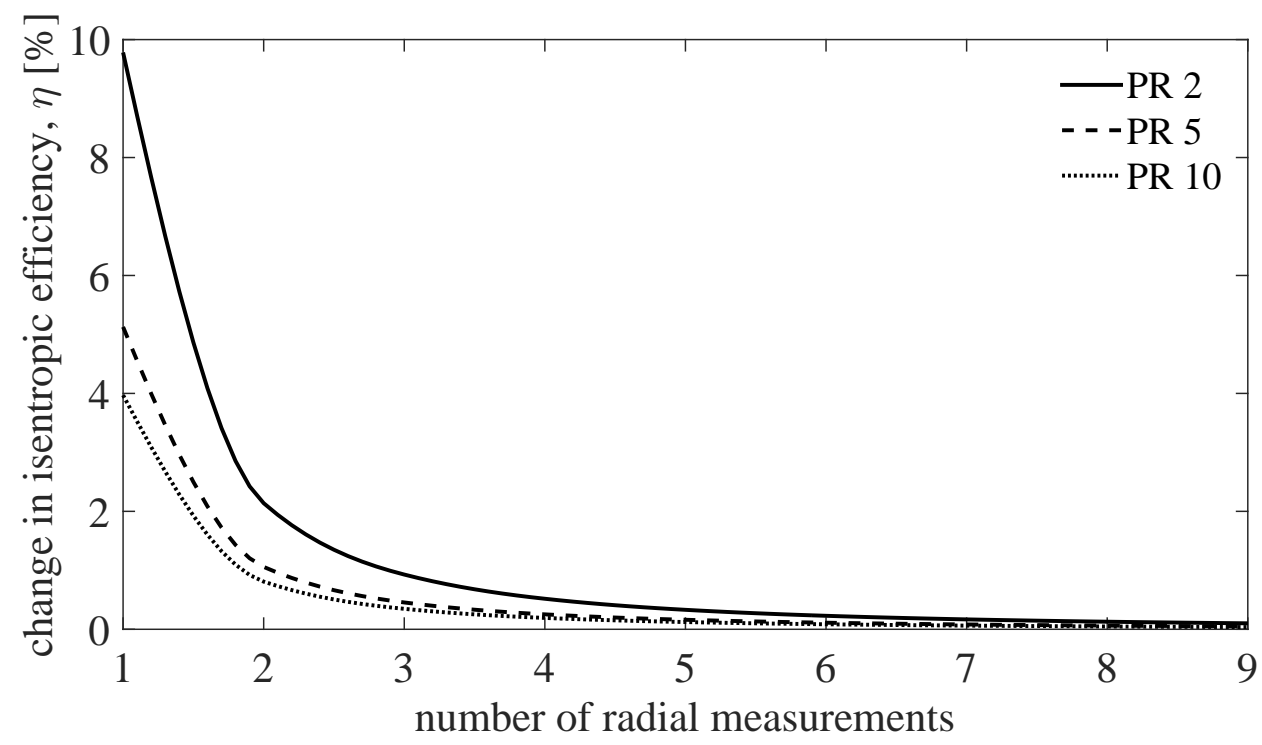

Figure 2. A graph showing the change in calculated compressor isentropic efficiency with increasing numbers of radially distributed stagnation pressure and temperature measurements at a single circumferential location $\left(T_{o, \text { in }}=300 \mathrm{~K}, \eta_{p}=0.85\right)$. 
Combination probes for stagnation pressure and temperature measurements in gas turbine engines7

\section{Probe aerodynamic calibration facility}

The Loughborough University probe aerodynamic calibration facility has been developed to allow the performance of stagnation pressure and temperature probes to be assessed under engine representative Mach number conditions. The facility is serviced by two Kaeser screw compressors that can pressurise up to $1 \mathrm{~kg} / \mathrm{s}$ of ambient air to $14 \mathrm{bar}$. Air delivered from the compressors is stored in a $100 \mathrm{~m}^{3}$ receiver tank that acts as a buffer to damp any pressure fluctuations. A 6" diameter pipeline transports compressed air from the receiver tank to the laboratory housing the test facility. This pipeline includes two in-series control valves that regulate the flow from the 14 bar supply pressure down to the target working section delivery pressure $(\leq 8 \mathrm{bar})$. The first (coarse) stage of pressure control is provided by a Severn Glocon piston actuated valve that instigates the bulk of the flow pressure drop required. A Spirax Sarco globe valve then refines the flow pressure to within $1 \%$ of the target value, providing the second (fine) stage of pressure control. Inside the laboratory, the $6 "$ pipeline delivers the regulated air to a convergent nozzle with a $60 \mathrm{~mm}$ throat diameter. This nozzle issues directly into the laboratory, forming a continuous-running free-jet with velocities up to Mach 1.0. A schematic diagram of the facility is shown in Figure 3 ,

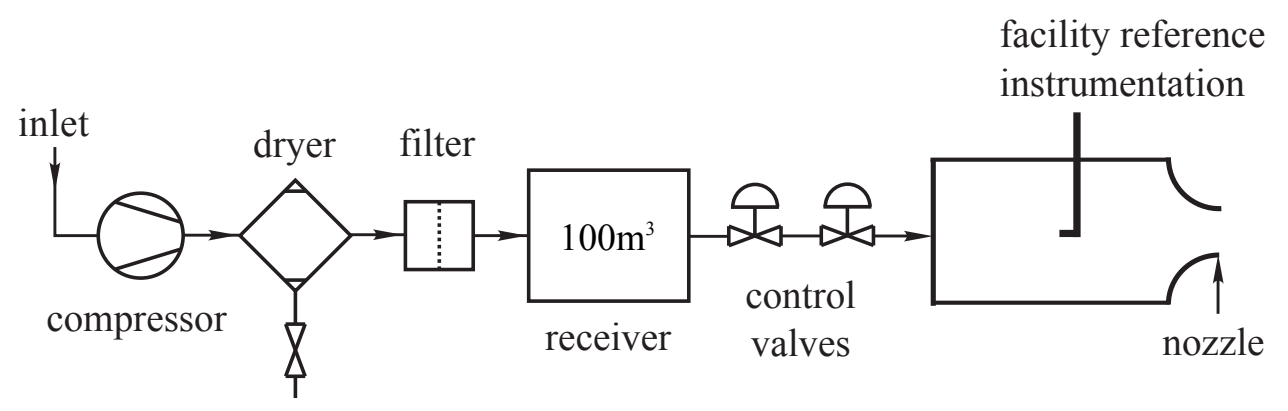

Figure 3. A schematic diagram of the probe aerodynamic calibration facility.

\subsection{Facility reference instrumentation}

The facility is equipped with reference instrumentation that monitors the free-stream stagnation pressure and temperature of the flow, as well as the laboratory static pressure. These measurements are used to determine conditions in the jet (e.g. Mach number) using isentropic flow equations. The static pressure is measured at a location downstream of the nozzle and away from the expanding jet using a Huba Controls 691 series absolute pressure transducer with the sensing port open to atmosphere. The stagnation pressure and temperature are measured at a common location within the 6 " diameter compressed air pipeline. In this pipeline, flow velocities are sufficiently low $(\leq$ Mach 0.15) that stagnation quantities can be detected. The stagnation temperature is measured using a passively ventilated thin-film PRT probe, connected in 4-wire mode to a Pico PT-104 resistance measuring instrument. The stagnation pressure is measured 
Combination probes for stagnation pressure and temperature measurements in gas turbine engines 8

using a Pitot tube, connected to a second Huba Controls 691 series absolute pressure transducer via a length of flexible tubing.

Prior to use in the facility, all reference instrumentation is statically calibrated against an appropriate traceable standard to reduce systematic uncertainties in the measurements. For atmospheric measurement conditions and a jet Mach number of 0.75 , the expanded uncertainties in the reference pressure and temperature measurements are 2.1 mbar and $0.09 \mathrm{~K}$ respectively (at $95 \%$ confidence). These values are based on systematic uncertainties associated with the static calibration equipment and installation environment, as well as random uncertainties determined from the standard deviation of samples of repeat measurements. Table 1 provides a detailed break down of these uncertainties.

\subsection{Probe under test}

The probe under test is positioned on the jet centreline at a distance $60 \mathrm{~mm}$ downstream of the nozzle throat. At this location, the probe recovery performance can be investigated by monitoring changes in indicated pressure and temperature at different free-stream Mach number conditions. The probe indicated temperature is measured using the same Pico PT-104 resistance measuring device that is used for stagnation temperature measurements. The probe indicated pressure is measured relative to the flow stagnation pressure using a Sensor Technics BTE5000 differential pressure transducer. Like the reference instrumentation, the probe under test is statically calibrated against traceable standards prior to installation in the facility. At atmospheric conditions with a jet Mach number of 0.75 , the expanded uncertainties in the probe measured pressure and temperature are 0.1 mbar and $0.02 \mathrm{~K}$ respectively (at $95 \%$ confidence). The random and systematic components of these uncertainties are detailed in Table 1 .

Table 1. Aerodynamic calibration uncertainties at atmospheric conditions for a jet Mach number of 0.75 (at $95 \%$ confidence).

\begin{tabular}{l|c|c|c} 
variable & systematic uncertainty & random uncertainty & expanded uncertainty \\
\hline$T_{o, \infty}(\mathrm{K})$ & 0.09 & 0.01 & 0.09 \\
$P_{o, \infty}(\mathrm{mbar})$ & 0.3 & 2.0 & 2.0 \\
$T_{\text {ind }}(\mathrm{K})$ & 0.02 & 0.01 & 0.02 \\
$P_{\text {ind }}(\mathrm{mbar})$ & 0.01 & 0.1 & 0.1 \\
$R_{p, T}$ & 0.003 & 0.001 & 0.003 \\
$R_{p, P}$ & 0.00001 & 0.0002 & 0.0002 \\
$M_{\infty}$ & 0.0003 & 0.003 & 0.003
\end{tabular}


Combination probes for stagnation pressure and temperature measurements in gas turbine engines9

\subsection{Probe recovery performance}

The recovery performance of the probe under test is quantified using pressure (eq. 4) and temperature (eq. 5) recovery factors, which are both functions of the dynamic properties of the flow:

$$
\begin{aligned}
R_{p, P} & =\frac{P_{i n d}-P_{\infty}}{P_{o, \infty}-P_{\infty}} \\
R_{p, T} & =\frac{T_{i n d}-T_{\infty}}{T_{o, \infty}-T_{\infty}}
\end{aligned}
$$

The recovery factors can be calculated using the probe indicated pressure and temperature, as well as knowledge of the jet flow conditions derived from reference instrumentation. Applying the Taylor series method of uncertainty propagation (Coleman \& Steele 1995), uncertainties in the pressure and temperature recovery factors are calculated as 0.0002 and 0.003 at a jet Mach number of 0.75 (at $95 \%$ confidence). These values vary with Mach number since the uncertainties represent greater proportions of the dynamic pressure and temperature at lower Mach numbers compared to higher Mach numbers. At a jet Mach number of 0.3 for example, the uncertainties in pressure and temperature recovery factor increase to 0.002 and 0.008 respectively. A similar trend is observed in jet Mach number, which is determined from the isentropic pressure relation. Using a Taylor series uncertainty propagation, the expanded Mach number uncertainty increases from 0.003 at Mach 0.75 to 0.008 at Mach 0.3 (at $95 \%$ confidence).

The pressure and temperature recovery factors characterise the ability of the probe under test to indicate the true stagnation properties of the flow. During gas turbine engine test campaigns, it is common to correct for any under-reads in probe indicated stagnation pressure and temperature using recovery factors determined via aerodynamic calibration. The corrected stagnation pressure and temperature are determined from eq. 6 and eq. 7 respectively.

$$
\begin{aligned}
P_{o, \infty} & =\frac{P_{i n d}+P_{\infty}\left(R_{p, P}-1\right)}{R_{p, P}} \\
T_{o, \infty} & =\frac{T_{i n d}+T_{\infty}\left(R_{p, T}-1\right)}{R_{p, T}}
\end{aligned}
$$

Figure 4 indicates how uncertainties in corrected stagnation pressures and temperatures are influenced by probe recovery factor. For recovery factors of unity, no post-test corrections are required hence the stagnation pressure and temperature uncertainties are solely governed by the accuracy of the respective measurement systems. For this example, this is assumed to be $\pm 0.05 \%$. For probe recovery factors below unity, stagnation pressure and temperature uncertainties rise due to additional questions over the accuracy of post-test corrections. This is influenced by uncertainties in the probe recovery factors and the static properties of the flow, which for this example are based on the aerodynamic calibration uncertainties detailed in Table 1. These uncertainties 
are expected to be greater in an engine test environment, since inconsistencies with aerodynamic calibration conditions (e.g. flow incidence angle) can result in unaccounted changes to probe recovery factors. Similarly, static pressures and temperatures must be determined from the local Mach number, which may not be well characterised at the measurement location. In seeking to achieve high stagnation pressure and temperature accuracy, probe recovery factors close to unity are therefore desirable such that the uncertainty contributions from post-test corrections remain small.

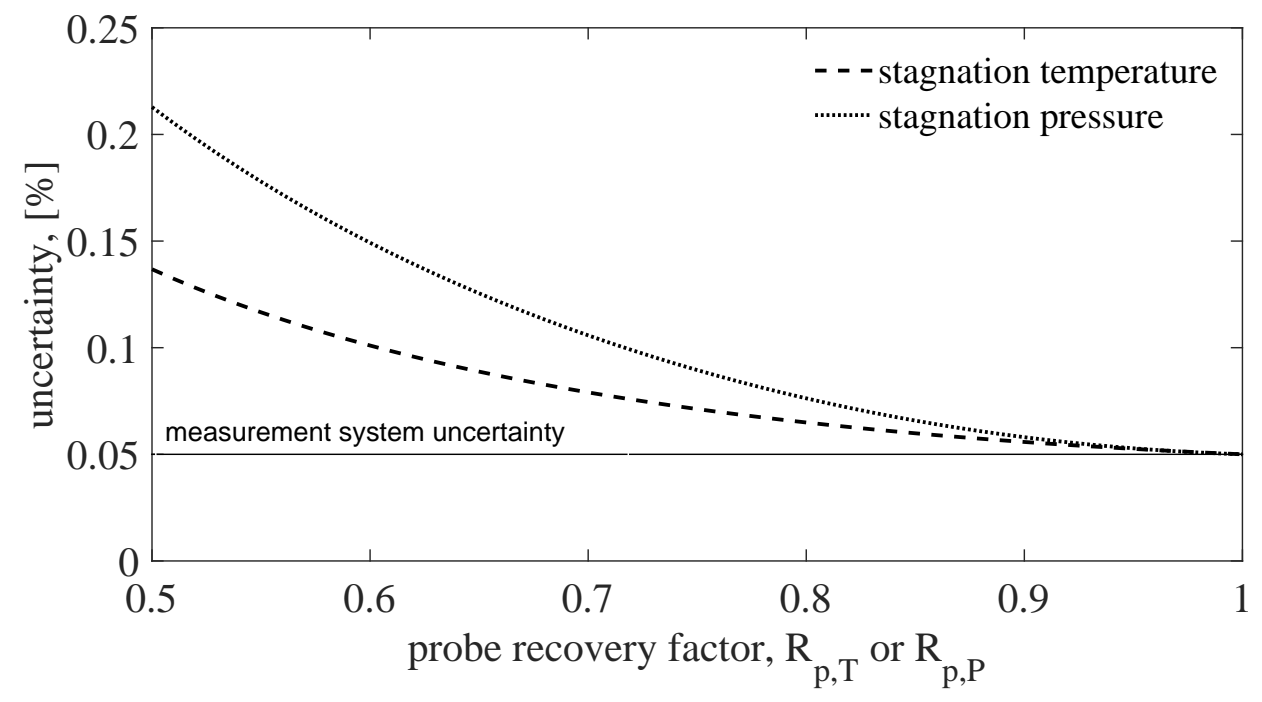

Figure 4. Graph showing uncertainties in stagnation pressure and temperature as a function of probe recovery factor. Uncertainties calculated using Taylor series propagations at atmospheric pressure and temperature conditions for a jet Mach number of 0.75 .

\section{Side-by-side combination probe}

The first combination stagnation pressure and temperature probe to be considered in this investigation is the side-by-side probe, which is shown in Figure 5. The probe is comprised of a thin-film platinum resistance thermometer (PRT) and a fine-bore pitot tube that are shielded within a common stagnation tube. The stagnation tube is constructed from a hollow stainless-steel cylinder, which contains a pair of rearward vent holes that promote a continuous flow of air through the device. At a given free-stream Mach number, the flow velocity within the stagnation tube is determined by the ratio of vent hole area to probe inlet area. This area ratio must be small enough to maintain sufficiently low velocities ( $\leq$ Mach 0.25 ) within the probe that stagnation properties can be measured (Markowski \& Moffatt 1948).

The side-by-side probe adopts a similar shield design to conventional Kiel-type probes that are routinely used in gas turbine engine testing. However, its novelty lies in the application of a thin-film PRT in place of a typical thermocouple sensor. 
Combination probes for stagnation pressure and temperature measurements in gas turbine engines11

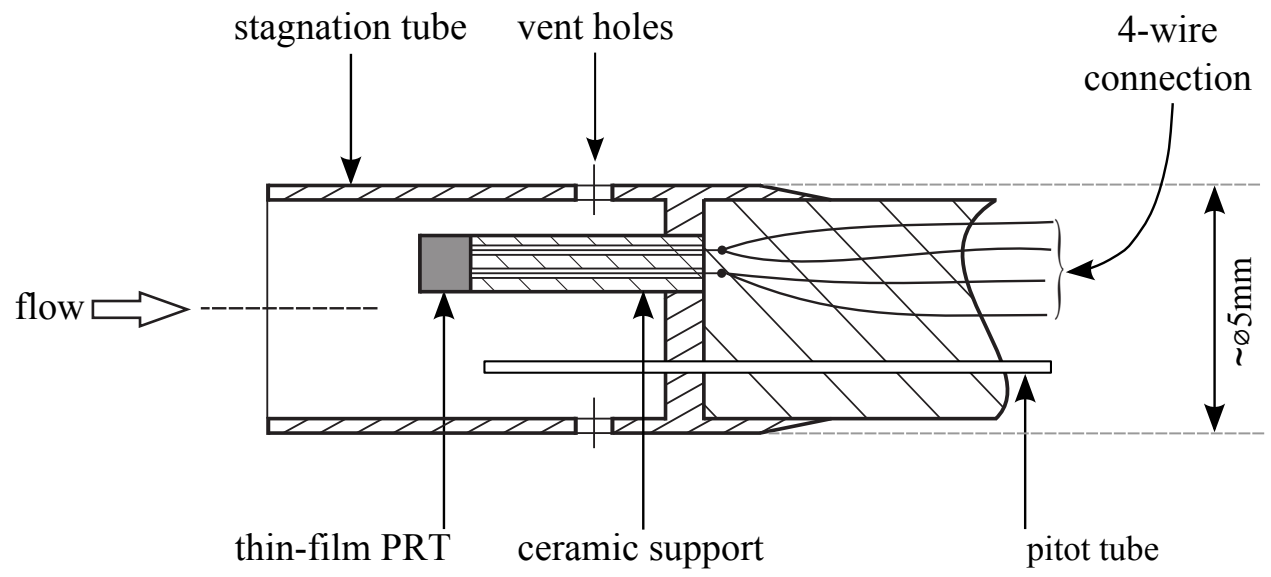

Figure 5. A schematic diagram of the side-by-side combined stagnation pressure and temperature probe.

This change is motivated by the superior temperature sensing accuracy offered by thinfilm PRTs at temperatures up to $750 K$ (Bonham, Thorpe, Erlund \& Stevenson 2013). Compared to thermocouples, thin-film PRTs have better static calibration retention and are less sensitive to disturbances from electrical noise. This is a consequence of the stable thermoelectric properties and high thermoelectric response of the platinum. Unlike thermocouples, thin-film PRTs also provide an absolute (rather than differential) temperature measurement, hence any uncertainties associated with reference junction temperature are eliminated. In the authors' experience, a temperature measurement uncertainty of $\pm 0.05 \%(0.15 K)$ can be achieved with PRTs at ambient temperature conditions, compared to $\pm 0.27 \%(0.81 K)$ with thermocouples. To obtain gas-path stagnation temperatures to an uncertainty of $\pm 0.1 \%$, the application of thin-film PRTs in preference to thermocouples is therefore clearly advantageous.

\subsection{Probe recovery performance}

Figure 6] shows pressure and temperature recovery factors for the side-by-side probe plotted as a function of free-stream Mach number. For comparison, Figure 7 also shows recovery characteristics for individual stagnation pressure and temperature Kiel probes, which represent the current standard for gas-path measurements. Both Kiel probes utilise passively ventilated cylindrical shields, with the pressure device incorporating a pitot tube and the temperature device employing a N-type thermocouple sensor.

Over the Mach number range considered, the pressure recovery factor of the sideby-side probe maintains a constant value of 0.99 . This is consistent with the pressure recovery characteristic shown for the conventional Kiel probe in Figure 7 , Such high pressure recovery performance implies that a minor post-test correction of 4 mbar is required at a Mach number of 0.75 and atmospheric pressure conditions. From Figure 4. the uncertainty associated with the corrected stagnation pressure at these conditions is clearly dominated by the performance of the pressure measuring system, with negligible 
Combination probes for stagnation pressure and temperature measurements in gas turbine engines 12

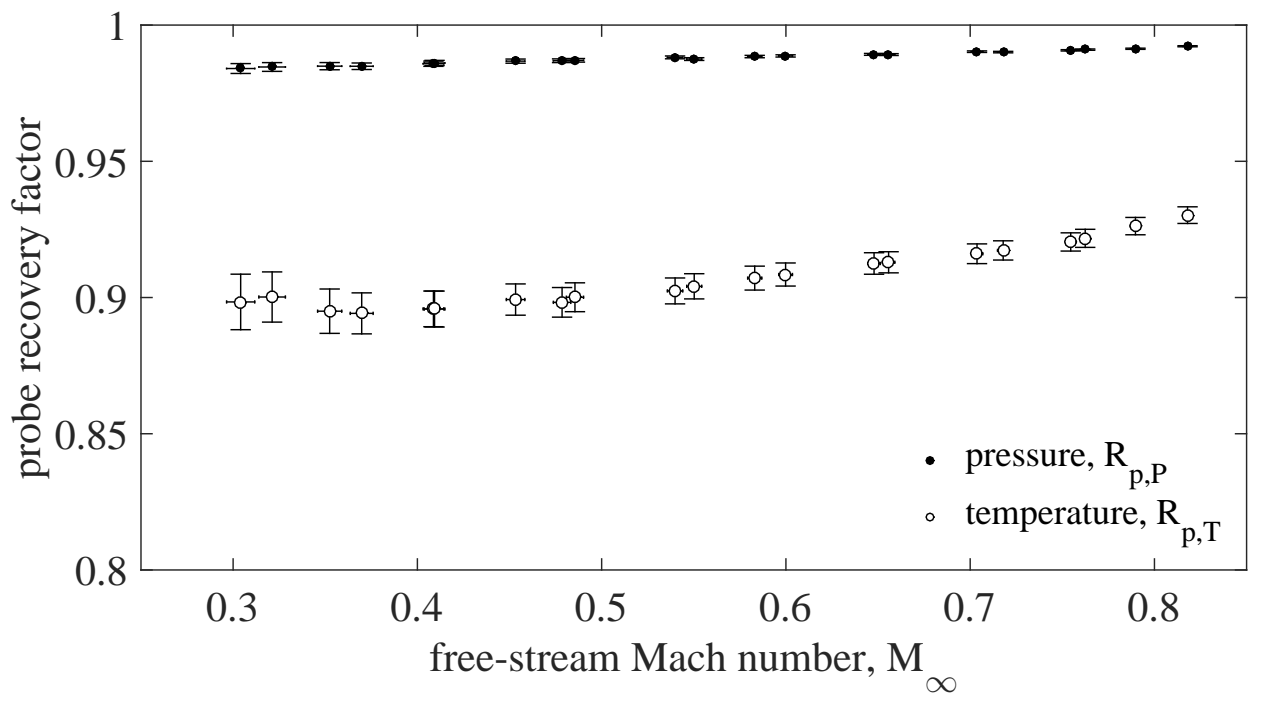

Figure 6. Graph showing the pressure and temperature recovery factor of the sideby-side probe as a function of free-stream Mach number.

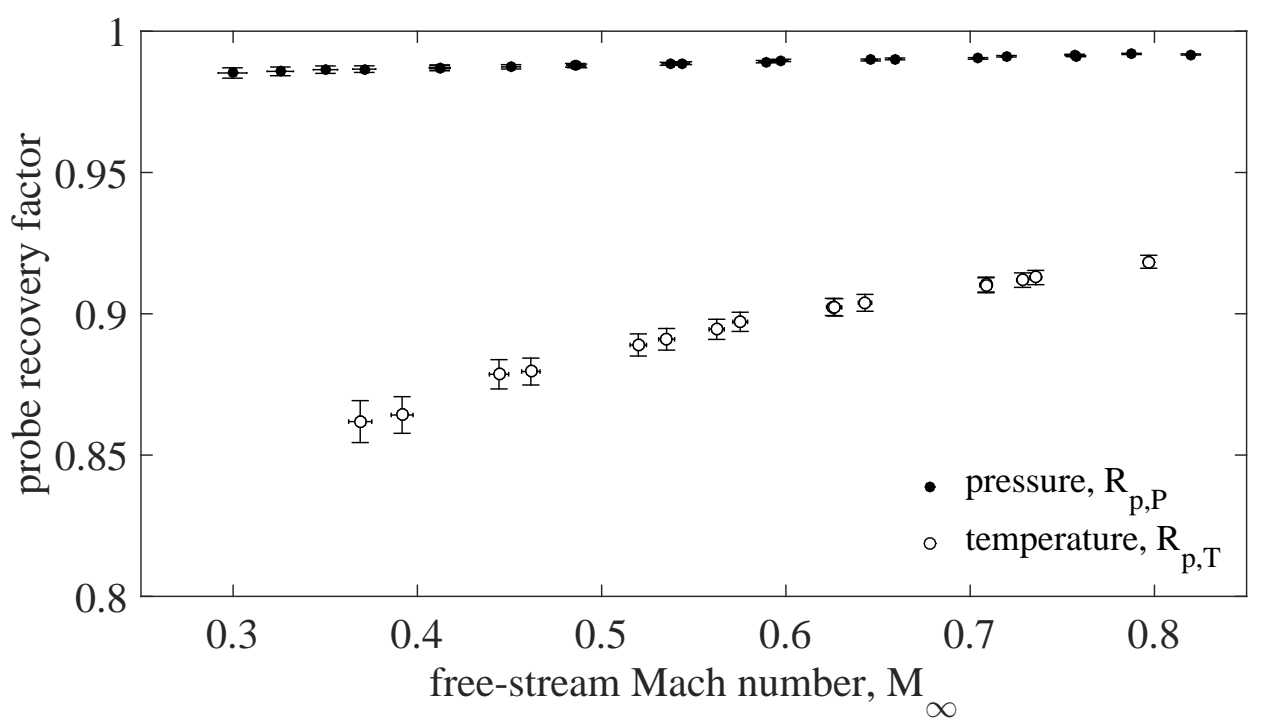

Figure 7. Graph showing pressure and temperature recovery factors for standard stagnation pressure and temperature Kiel probes as a function of free-stream Mach number.

contribution from the post-test correction. This is desirable since the uncertainty associated with post-test corrections may become large an engine test environment. For high stagnation pressure accuracy, both the side-by-side and standard Kiel probes therefore represent good candidate designs.

The temperature recovery factor of the side-by-side probe exhibits notable variation with Mach number, increasing from 0.89 to 0.93 across the range of conditions considered. This is comparable to the temperature recovery performance of the 
standard Kiel probe shown in Figure 7. Such variation is undesirable in an engine test environment, since uncertainties in the gas-path Mach number make the selection of an appropriate recovery factor challenging. The comparatively low temperature recovery performance also implies that a large post-test correction of $2.43 \mathrm{~K}$ is required at Mach 0.75 and atmospheric temperature conditions. From Figure 4 , it is apparent that the uncertainty associated with the corrected stagnation temperature at these conditions is influenced by the performance of the measuring system as well as the post-test correction. In striving to achieve high stagnation temperature accuracy, probes with higher and flatter recovery characteristics compared to the side-by-side probe must consequently be sought.

\section{Dual-skin combination probe}

Low probe temperature recovery factors can often be attributed to conductive heat losses from the temperature sensor to the surroundings (see for example Wilson et al,(2012)). These conductive losses are driven by a temperature difference between the near-stagnated air within the stagnation tube and the free-stream air passing over the external surfaces of the probe. The temperature indicated by the sensor is therefore below the stagnation temperature of the flow. Figure 8 shows the dual-skin combination probe, which uses a novel shield to reduce these unwanted conductive heat transfer effects and deliver high temperature recovery factors (Thorpe, Bonham \& Erlund 2016). Although the shield is more complex compared to standard Kiels, recent advances in additive manufacturing technology have expanded the design envelope to make such designs feasible.

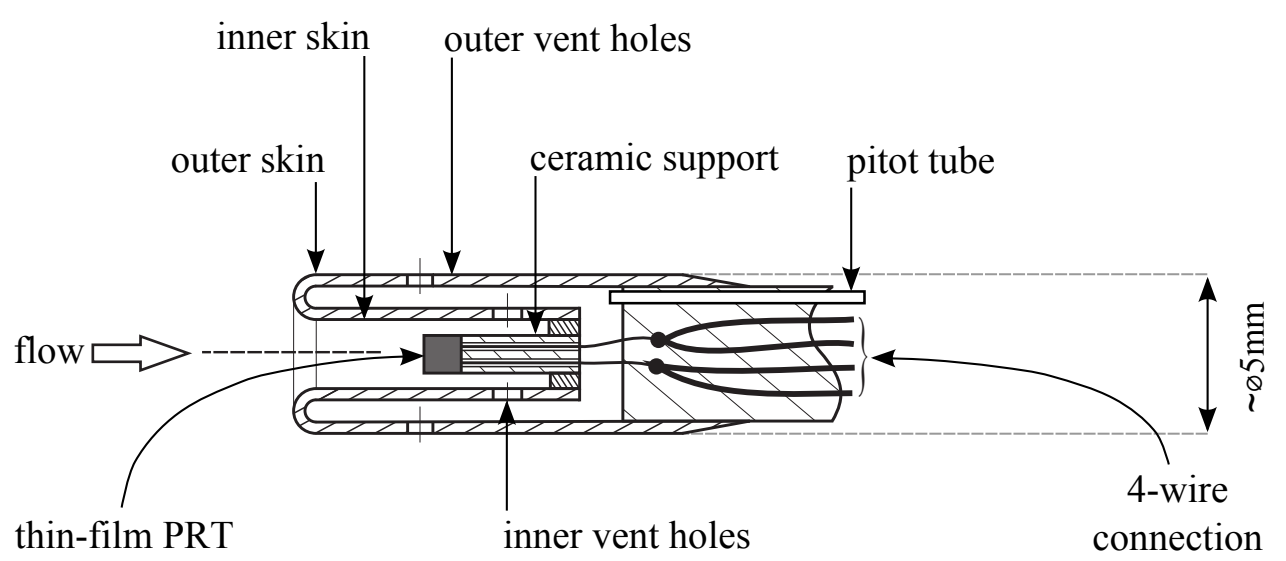

Figure 8. A schematic diagram of the dual-skin combined stagnation pressure and temperature probe.

The dual-skin probe is essentially comprised of two concentric stainless-steel cylinders, which each contain an array of vent holes. The external cylinder forms the outer body of the probe and is exposed to the free-stream flow conditions. The internal cylinder acts as the stagnation tube and houses the thin-film PRT sensor used 
for stagnation temperature measurements. The two cylinders are separated by a radial gap, which forms an annular passage inside the probe. This passage is continuously purged with low velocity air $(\leq$ Mach 0.25$)$ from the stagnation tube, which has a pressure and temperature close to the stagnation values. This acts to thermally isolate the sensor from the cooler external surfaces of the probe, reducing conductive heat losses.

The annular passage also provides a suitable location for the installation of a finebore pitot tube for the measurement of stagnation pressure. Unfortunately, as air travels through the probe viscous effects will cause some of the inlet stagnation pressure to be lost. This loss occurs as a consequence of friction as air passes over the solid surfaces of the probe, as well as due to separation as air passes through the two vent hole arrays. Since the internal surfaces of the probe have low wetted area, the pressure loss due to frictional effects will be small. The dominant pressure loss will therefore be associated with flow through the two vent hole arrays. For the probe to provide an accurate indication of stagnation pressure, it is the pressure loss across the internal vent holes upstream of the pitot tube that must be minimised. In practice, this requires the provision of a high ratio of internal vent hole area to probe inlet area, as well as a low stagnation tube velocity. The latter is dominated by the ratio of external vent hole area to probe inlet area for a given Mach number condition. In the design of the dual-skin combination probe, the area ratios between the two vent hole arrays and the inlet must therefore be carefully selected.

\subsection{Vent hole arrays}

Figures 9 and 10 show pressure and temperature recovery factors obtained from six dual-skin probes constructed with different internal vent hole areas, but fixed inlet and external vent hole areas. As the ratio $A_{\text {internal }} / A_{\text {inlet }}$ is increased, the pressure loss through the vent holes reduces and hence the probe pressure recovery factor is improved. This trend persists up to an area ratio of 6.1 , at which the pressure recovery factor attains a maximum value of 0.98 . Over the range of area ratios investigated, the probe temperature recovery factor remains approximately constant. This suggests that there is little variation in conditions within the stagnation tube and temperature recovery effects of the flow over the sensor therefore remain unchanged. As a consequence, the ratio of internal vent hole area to probe inlet area can be selected to maximise pressure recovery performance, without compromising temperature recovery performance.

Figures 11 and 12 show pressure and temperature recovery factors obtained from seven dual-skin probes with different external vent hole areas, but fixed inlet and internal vent hole areas. As the ratio $A_{\text {external }} / A_{\text {inlet }}$ is reduced, the velocity through the stagnation tube decreases and hence the pressure loss through the internal vent holes also drops. At an area ratio of 0.2 , the probe pressure recovery factor attains a maximum value of 0.98. At this area ratio, a maximum temperature recovery factor of 0.96 is attained. This is also a consequence of the decrease in velocity through the 
Combination probes for stagnation pressure and temperature measurements in gas turbine engines 15

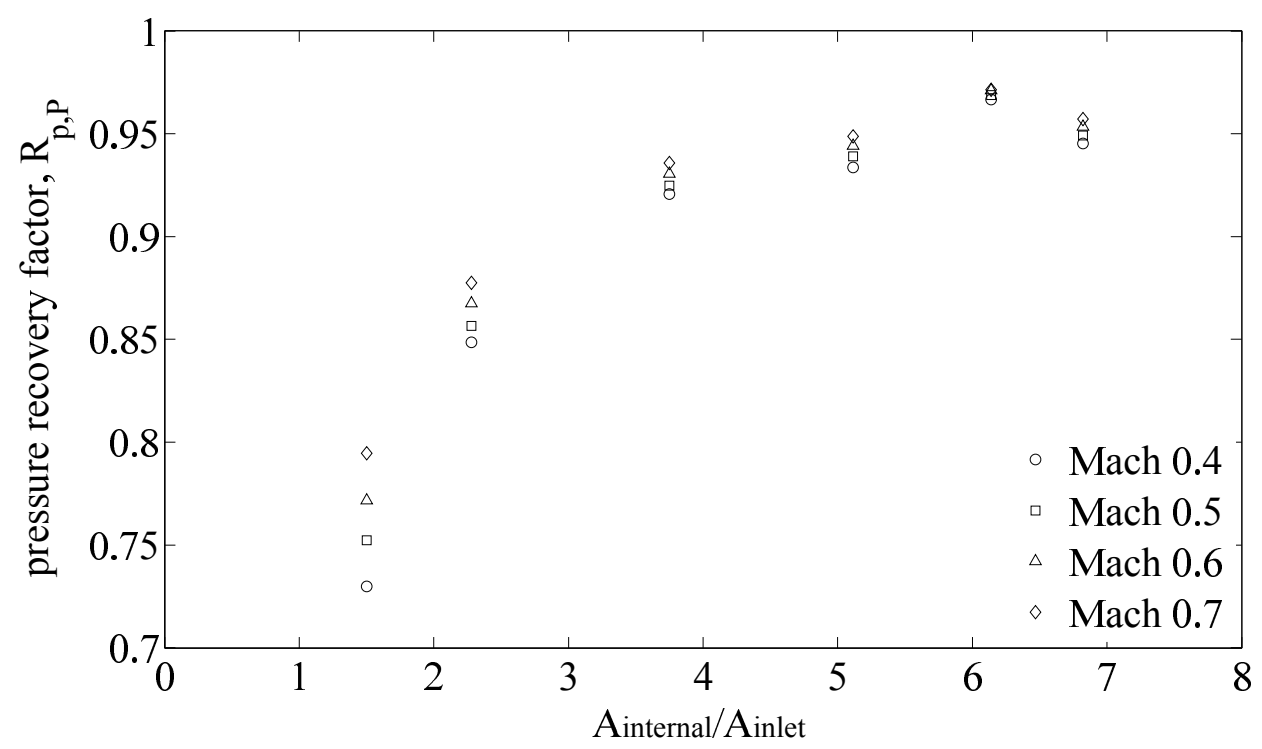

Figure 9. Pressure recovery factor of dual-skin probes with different internal vent hole areas $\left(A_{\text {external }} / A_{\text {inlet }}=0.38\right)$.

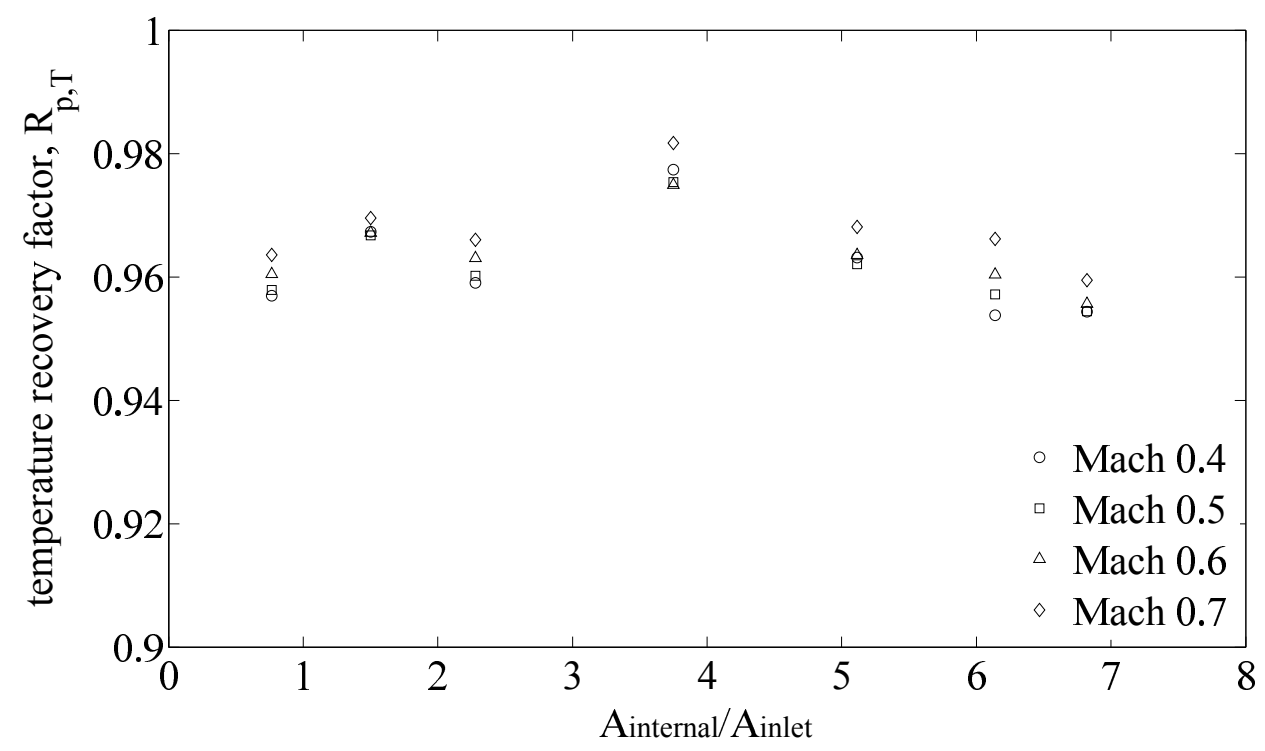

Figure 10. Temperature recovery factor of several dual-skin probes with different internal vent hole areas $\left(A_{\text {external }} / A_{\text {inlet }}=0.38\right)$.

stagnation tube, which reduces temperature recovery effects of the flow over the sensor. The optimum ratio of internal vent hole area to probe inlet area can therefore be selected to maximise both pressure and temperature recovery performance. 
Combination probes for stagnation pressure and temperature measurements in gas turbine engines16

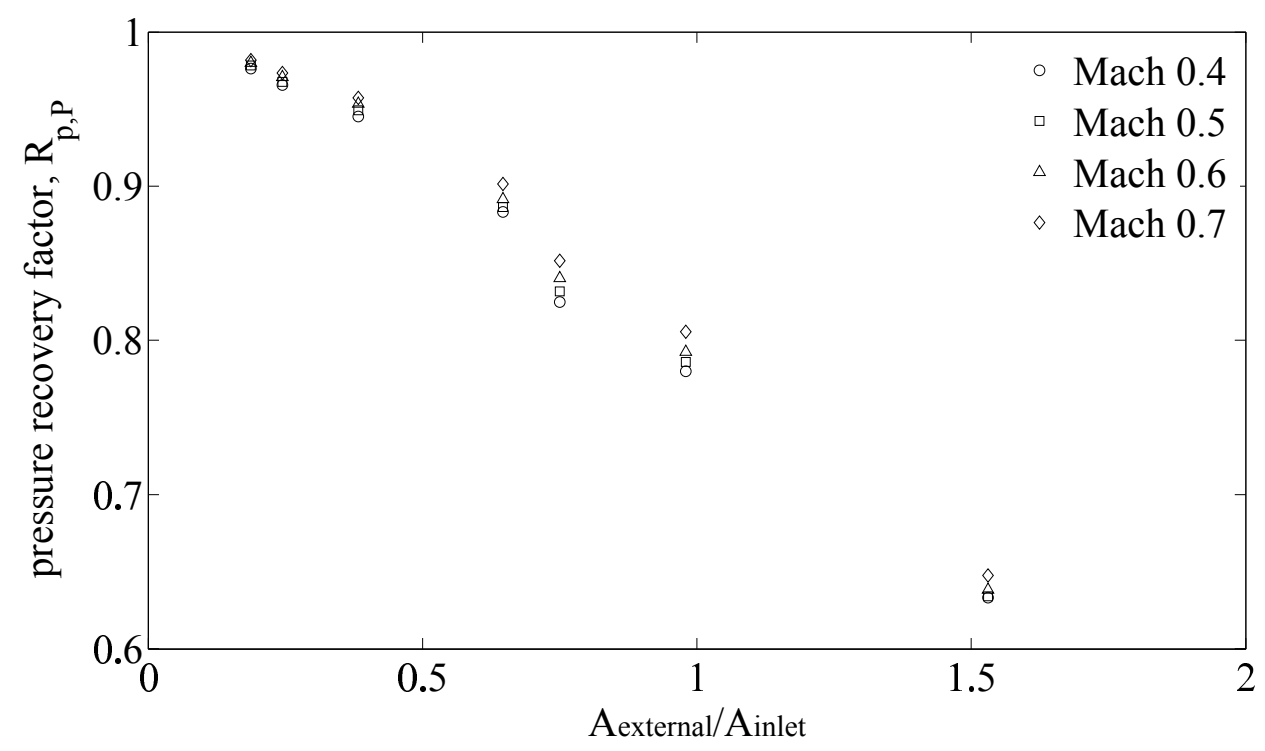

Figure 11. Pressure recovery factor of several dual-skin probes with different external vent hole areas $\left(A_{\text {internal }} / A_{\text {inlet }}=6.82\right)$.

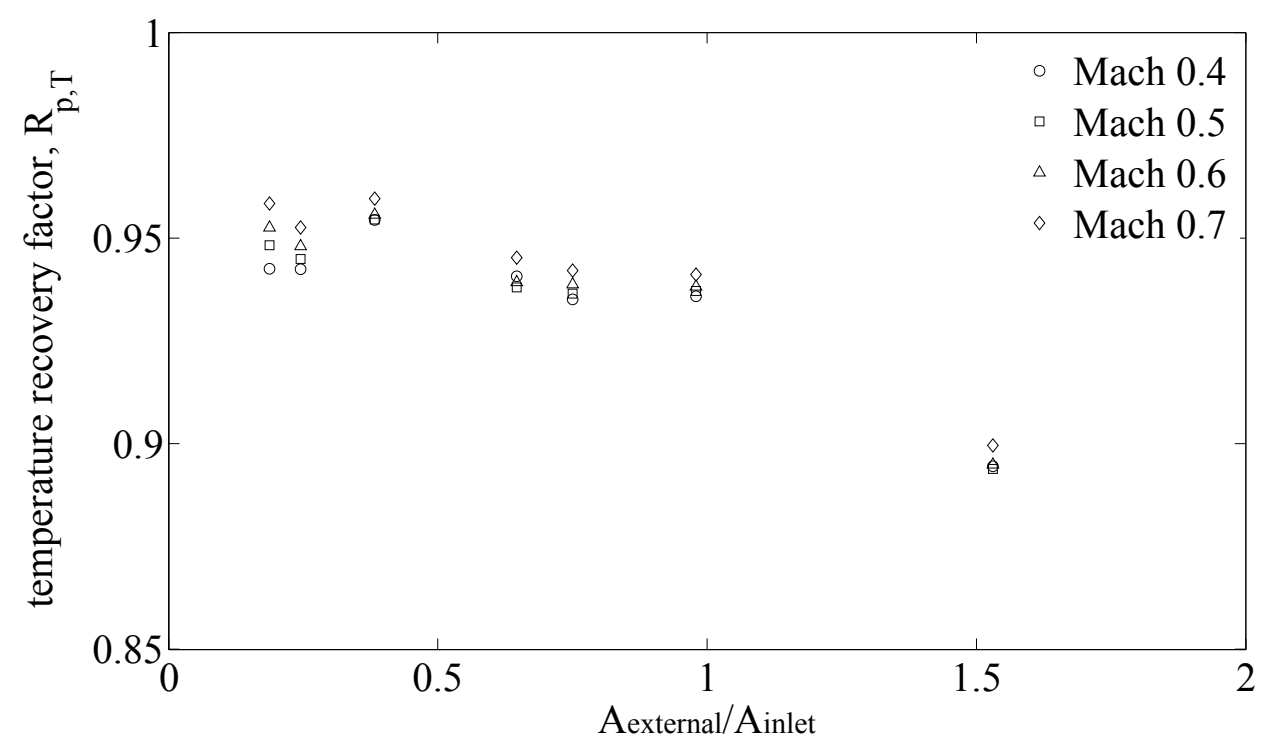

Figure 12. Temperature recovery factor of several dual-skin probes with different external vent hole areas $\left(A_{\text {internal }} / A_{\text {inlet }}=6.82\right)$.

\subsection{Probe recovery performance}

Figures 13 and 14 show the pressure and temperature recovery performance of an optimised dual-skin combination probe constructed with internal and external vent area ratios of 6.82 and 0.38 respectively. Also plotted for comparison are pressure and temperature recovery factors for the side-by-side probe, which are taken from Figure 6. 
Combination probes for stagnation pressure and temperature measurements in gas turbine engines 17

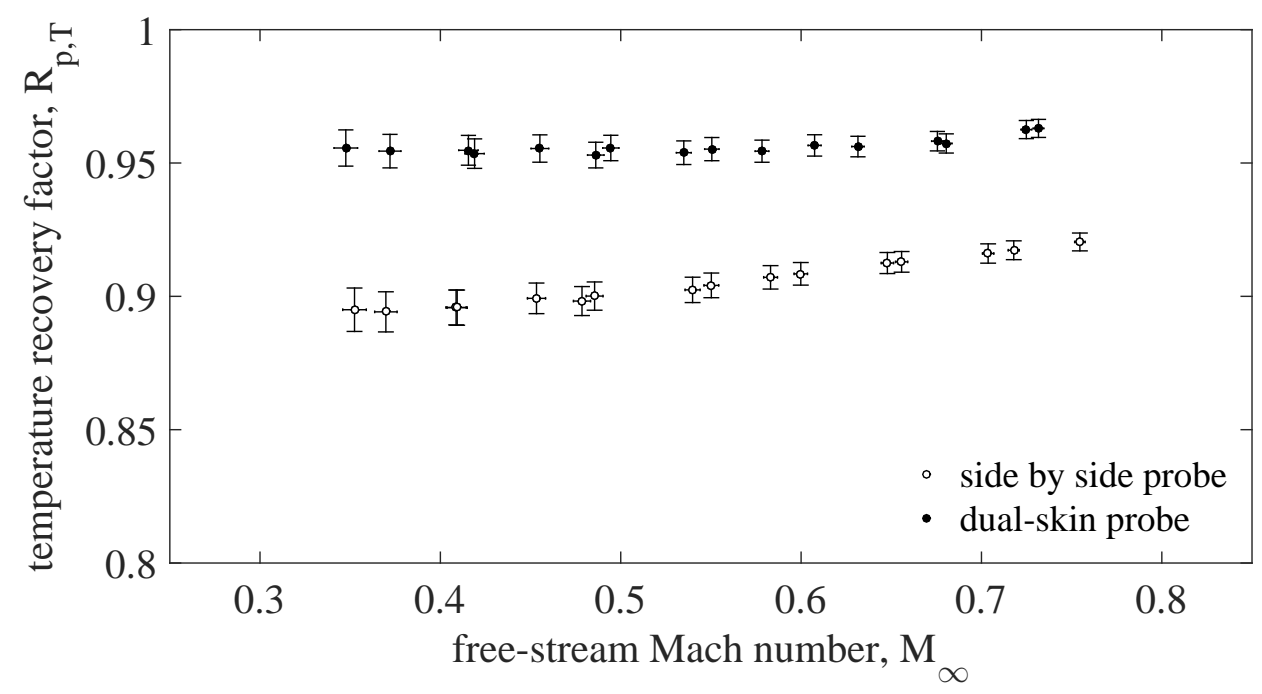

Figure 13. A graph showing the temperature recovery performance of the optimised dual-skin probe compared to the side-by-side probe.

The temperature recovery factor of the dual-skin probe maintains a constant value of 0.96 over the Mach number range investigated. Compared to the side-by-side probe, this improved performance is attributed to a reduction in conductive heat transfer away from the temperature sensor, which is facilitated by the double-walled probe design. At Mach 0.75 and atmospheric temperature conditions, the dual-skin probe requires a posttest correction of $1.20 \mathrm{~K}$, which is approximately half of the $2.43 \mathrm{~K}$ correction needed for the side-by-side probe. Referring back to Figure 4, the uncertainty in the corrected stagnation temperature will therefore reduce due to a smaller contribution from the post-test correction. To achieve high stagnation temperature accuracy, the adoption of the dual-skin probe in preference to the side-by-side probe is therefore considered advantageous.

Over the Mach number range investigated, a pressure recovery factor of 0.95 is recorded for the dual-skin probe, compared to a value of 0.99 for the side-by-side probe. These values correspond to stagnation pressure corrections of 21 mbar and 4 mbar respectively (at Mach 0.75 and atmospheric pressure conditions). For the dual-skin probe, the comparatively larger post-test correction is attributed to the loss of stagnation pressure as air travels through the complex passages inside the probe head. These losses are diminished in the side-by-side probe since the air is required to traverse a much simpler route. In the case of the dual-skin probe, the uncertainty in the corrected stagnation pressure will therefore increase due to the contribution of the post-test correction (see Figure 4). In order to achieve the highest levels of accuracy, an alternative probe design that combines the high temperature recovery performance of the dual-skin probe and the high pressure recovery performance of the side-by-side probe is consequently required. 
Combination probes for stagnation pressure and temperature measurements in gas turbine engines18

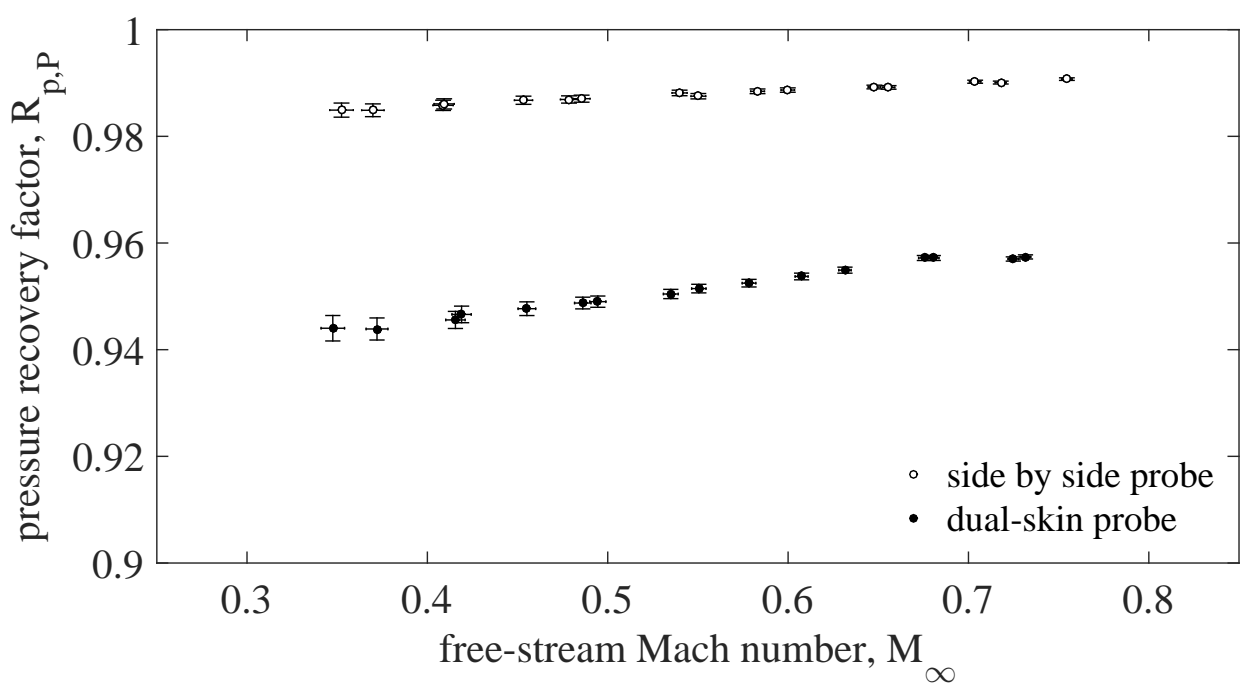

Figure 14. A graph showing the pressure recovery performance of the optimised dual-skin probe compared to the side-by-side probe.

\section{Concentric combination probe}

The concentric probe shown in Figure 15 is the final combination stagnation pressure and temperature probe to be considered in this investigation. Like the dual-skin probe, the concentric probe is intended to limit conductive heat loss from the thin-film PRT sensor by thermally isolating it from the cool external surfaces of the probe. This is achieved by adopting a new arrangement of concentric cylinders, in which the inner cylinder is mounted to the outer cylinder using three radial tubes. These tubes comprise the vent holes from the inner cylinder and are therefore continuously purged with low velocity air close to the stagnation temperature. As a result, any conduction between the inner and outer cylinders is minimised. In the concentric design, the stagnation pressure is measured in the outer cylinder via a fine-bore pitot tube. Compared to the dual-skin probe, this arrangement is less susceptible to upstream stagnation pressure losses since the only viscous effects arise from friction along the cylinder walls. More accurate indications of stagnation pressure should therefore be achievable.

\subsection{Probe recovery performance}

Pressure and temperature recovery factors for the concentric combination probe are shown in Figures [16 and 17, alongside corresponding data for the side-by-side and dual-skin combination probes. The pressure recovery performance of the concentric probe is consistent with that of the side-by-side probe, exhibiting a recovery factor of 0.99 over the Mach number range investigated. In comparison to the dual-skin probe, a smaller post-test correction is consequently required (4 mbar at Mach 0.75 and atmospheric conditions) and the uncertainty in the corrected stagnation temperature reduced. At this level of pressure recovery performance, it is implied from Figure 4 that 
Combination probes for stagnation pressure and temperature measurements in gas turbine engines19

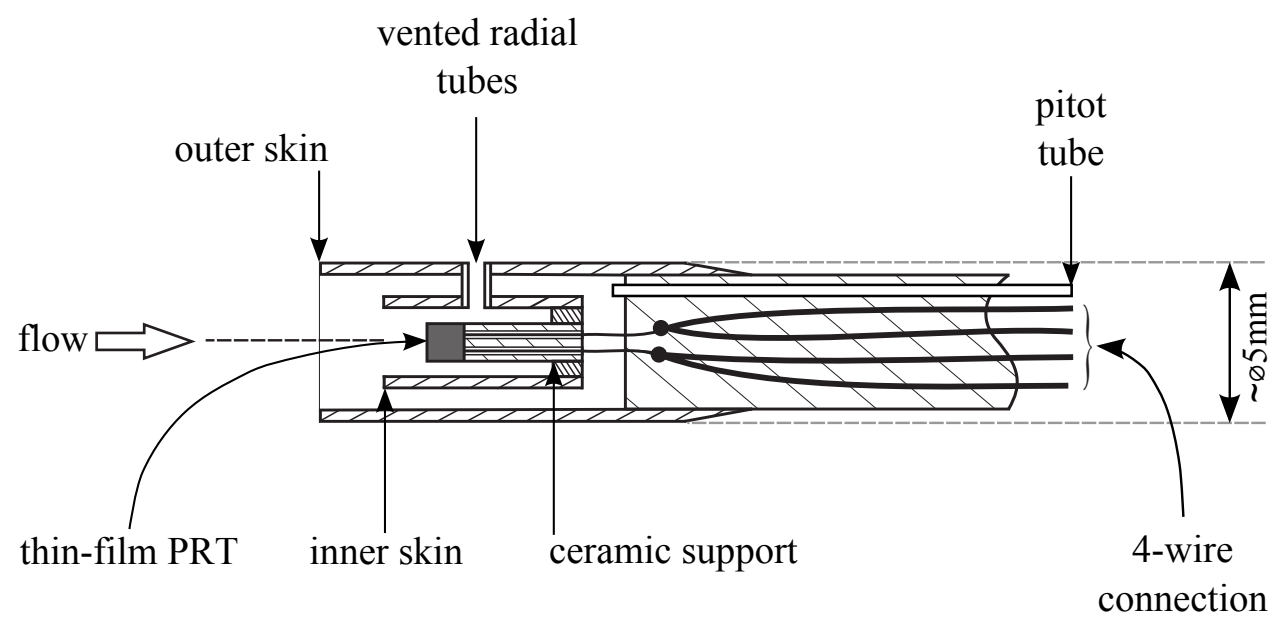

Figure 15. A schematic diagram of the concentric combined stagnation pressure and temperature probe.

an uncertainty approaching the performance of the pressure measuring system can be achieved. The temperature recovery performance of the concentric probe is slightly lower than that of the dual-skin probe, with a recovery factor of 0.95 recorded at a free-stream Mach number of 0.75 . This corresponds to a necessary post-test correction of $1.52 \mathrm{~K}$ at atmospheric temperature conditions. Although this level of correction is slightly higher compared to the dual-skin probe $(1.20 \mathrm{~K})$, the attendant increase in stagnation temperature uncertainty implied in Figure 4 is small. In terms of both stagnation pressure and temperature accuracy, the concentric probe is therefore considered to offer the best combination of recovery performance.

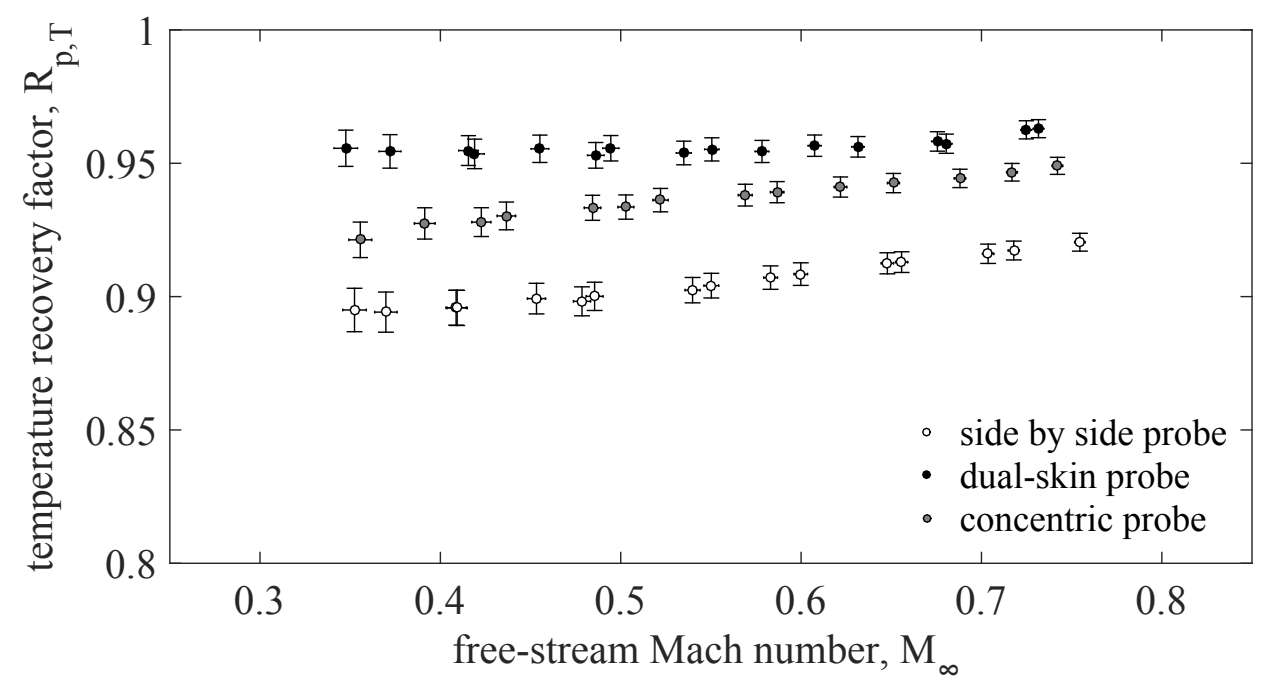

Figure 16. A graph showing the temperature recovery performance of the concentric probe compared to the optimised dual-skin and side-by-side probes.

Figure 18 compares the performance of the concentric combination probe against 
Combination probes for stagnation pressure and temperature measurements in gas turbine engines20

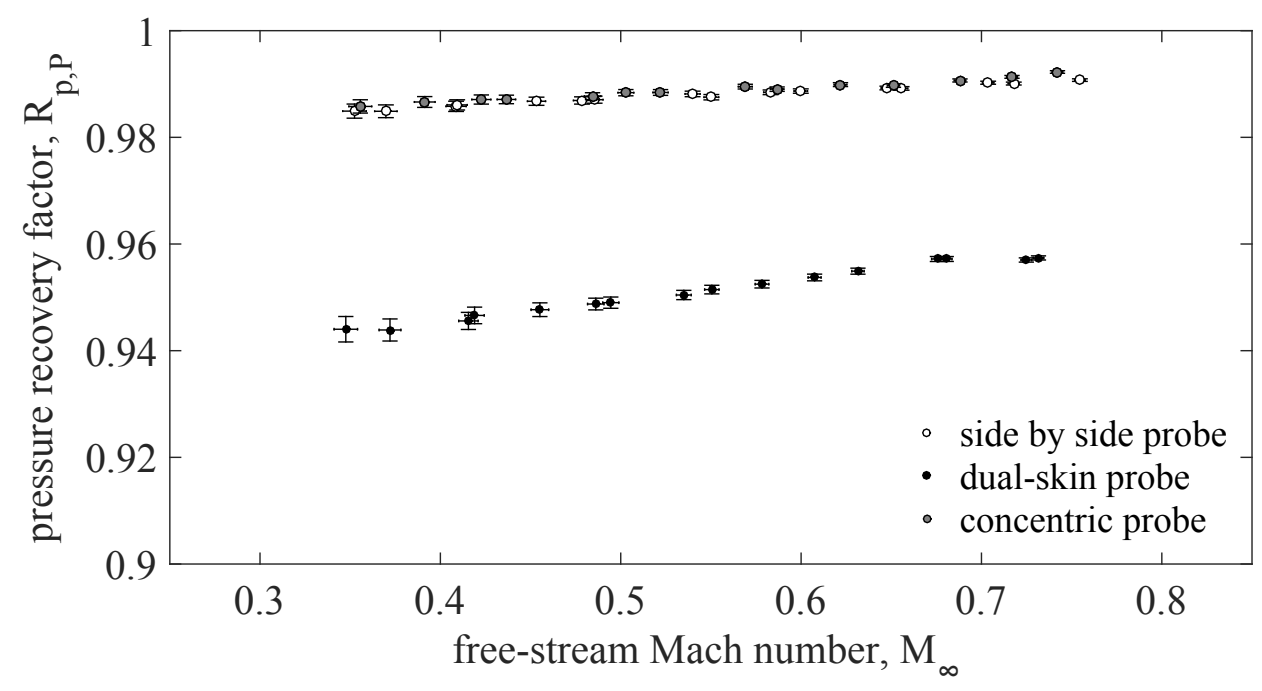

Figure 17. A graph showing the pressure recovery performance of the concentric probe compared to the optimised dual-skin and side-by-side probes.

the performance of the standard Kiel probes from Figure 7 by considering the impact of uncertainties in stagnation pressure and temperature on calculated compressor efficiency. This takes into account the uncertainties that have previously been quoted for pressure and temperature measuring systems, as well as uncertainties associated with post-test corrections that are derived from the aerodynamic calibration uncertainties in Table 1 . Although the latter uncertainties are likely to be higher in an engine test environment, this represents the best available data.

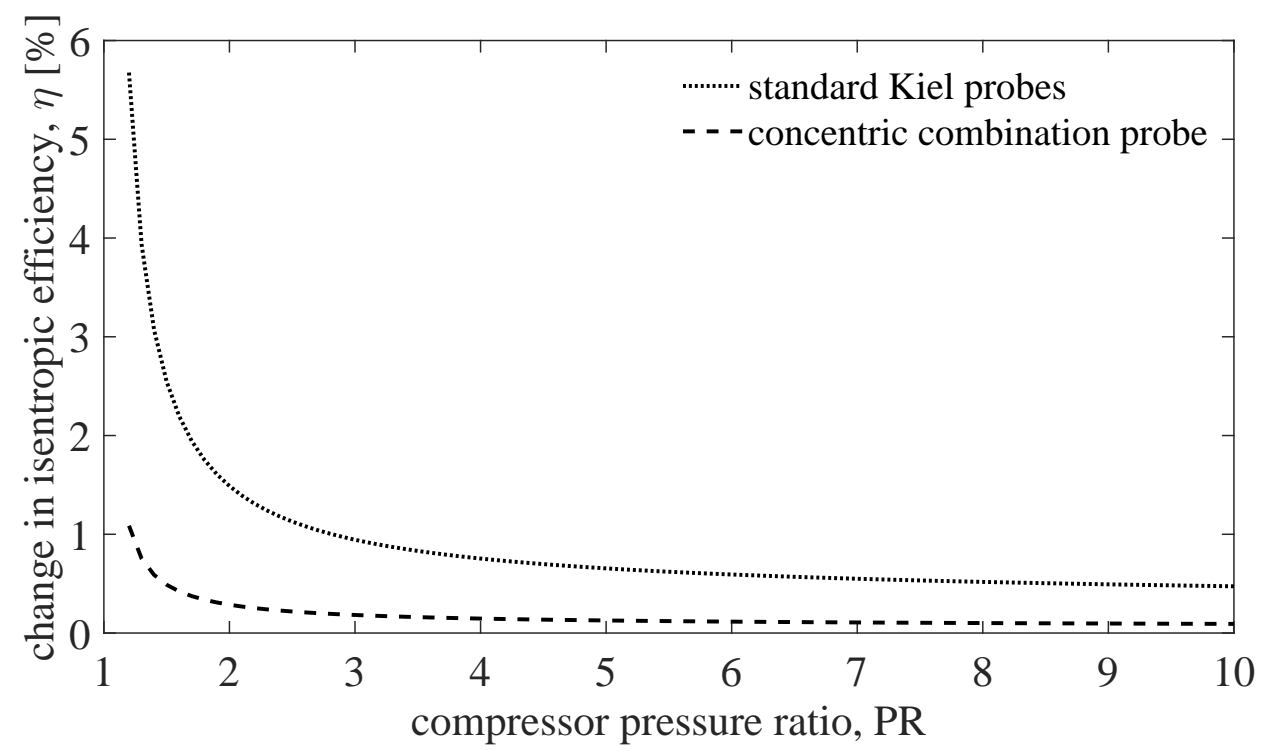

Figure 18. A graph comparing uncertainties in the calculated isentropic efficiency of a compressor for standard Kiel probes and the new concentric combination probe $\left(T_{o, \text { in }}=300 K, \eta_{p}=0.85\right)$. 
Combination probes for stagnation pressure and temperature measurements in gas turbine engines21

From Figure 18, it is clear that standard Kiel probes cannot achieve the target $\pm 1 \%$ uncertainty in compressor efficiency for pressure ratios $\leq 3$. This is due to a combination of poor thermocouple sensing accuracy and low probe temperature recovery performance, which result in stagnation temperature uncertainties of $\pm 0.3 \%$ at $300 \mathrm{~K}$. These uncertainties reduce with increasing temperature, but only achieve values within $\pm 0.1 \%$ at temperatures above $800 K$. In contrast, the concentric probe satisfies the compressor uncertainty target for all pressure ratios $\geq 1.2$. This is attributed to improved measurement accuracy offered by the thin-film PRT sensor as well as the higher probe temperature recovery performance, which together reduce stagnation temperature uncertainties close to $\pm 0.05 \%$ at $300 \mathrm{~K}$. At the maximum sensor temperature of $750 \mathrm{~K}$, this reduces further to $\pm 0.03 \%$. Compared to standard Kiels, the concentric probe is therefore offers particular advantages for applications in low pressure ratio components in the cold end of the engine. For hotter and higher pressure ratio components, the concentric probe continues deliver improved accuracy compared to standard Kiel probes. However, the limited sensor temperature rating practically prohibits its use beyond the intermediate pressure compression system. Prior to employing the concentric probe in these applications it would be prudent to assess its sensitivity to flow incidence angle, since the flow direction may not always be well-aligned with the axis of the probe. However, previous work has suggested that probe designs with low susceptibility to conductive heat loss have a greater range of angular insensitivity compared to designs associated with high conductive losses (Bonham et al. 2013). This is because the probes are less responsive to variations in shield temperature, caused by changes in the external flow field around the probe.

\section{Conclusion}

This article considers three new combination probes that deliver lower levels of uncertainty compared to the individual Kiel probes that are commonly used for steadystate gas-path stagnation pressure and temperature measurements. For a given flow path blockage, the combination probes are able to provide up to twice the spatial measurement resolution compared to individual Kiel probes. This allows more accurate area-weighted averages of stagnation pressure and temperature to be determined across the gas-path. To achieve high point measurement accuracy, the probes use thin-film PRTs in preference to conventional thermocouple sensors due to their comparatively lower measurement uncertainty for applications up to $750 \mathrm{~K}( \pm 0.05 \%$ at $300 \mathrm{~K})$. Additionally, shields with high pressure and temperature recovery performance $\left(R_{p, T} \approx\right.$ $R_{p, P} \approx 1$ ) are employed such that low-levels of uncertainty are introduced by posttest corrections. Practically, this is achieved by reducing unwanted heat transfer from the temperature sensor and limiting stagnation pressure losses upstream of the pitot tube. Of the three devices considered here, the concentric probe provides the best combination of these design features. The probe attains stagnation pressure and temperature uncertainties close to $\pm 0.05 \%$ and consequently permits the assessment of 
turbomachinery component efficiencies to the target $\pm 1 \%$ over a wide range of pressure ratios. This represents an improvement relative to standard Kiel probes, which cannot achieve $\pm 1 \%$ in efficiency at low pressure ratios due to higher stagnation temperature uncertainties. On this basis, the concentric combination probe could successfully be adopted for measurements in low pressure ratio components in the cold end of the engine, where modest pressure and temperature changes make the realisation of the target accuracy most challenging. However, some further investigation could usefully be made of the angular sensitivity of the probe before it is employed in these applications.

\section{Nomenclature}

$A_{\text {inlet }} \quad$ area of probe inlet

$A_{\text {internal }}$ total area of internal vent hole array

$A_{\text {external }}$ total area of external vent hole array

$M_{\infty} \quad$ free-stream Mach number

$P_{\text {ind }} \quad$ probe indicated pressure

$P_{o, \text { in }} \quad$ compressor inlet stagnation pressure

$P_{o, \text { out }} \quad$ compressor outlet stagnation pressure

$P_{\infty} \quad$ free-stream static pressure

$P_{o, \infty} \quad$ free-stream stagnation pressure

$P R \quad$ pressure ratio

$P R_{\text {wall }} \quad$ pressure ratio at annulus wall

$r \quad$ annulus radius

$r_{i} \quad$ inner annulus radius

$r_{o} \quad$ outer annulus radius

$R_{p, P} \quad$ probe pressure recovery factor

$R_{p, T} \quad$ probe temperature recovery factor

$T_{\text {ind }} \quad$ probe indicated temperature

$T_{o, \text { in }} \quad$ compressor inlet stagnation temperature

$T_{o, \text { out }} \quad$ compressor outlet stagnation temperature

$T_{\infty} \quad$ free-stream static temperature

$T_{o, \infty} \quad$ free-stream stagnation temperature

$T R \quad$ compressor temperature ratio

$T R_{\text {wall }} \quad$ compressor temperature ratio at annulus wall

$U \quad$ expanded uncertainty

$\eta \quad$ compressor isentropic efficiency

$\eta_{p} \quad$ compressor polytropic efficiency

$\gamma \quad$ specific heat ratio 
Combination probes for stagnation pressure and temperature measurements in gas turbine engines 23

\section{References}

Agnew, B., Elder, R. \& Terrel, M. (1985), An investigation of the response of temperature sensing probes to an unsteady flow field, in 'ASME 1985 International Gas Turbine Conference and Exhibit', American Society of Mechanical Engineers, pp. V001T03A061-V001T03A061.

Allan, III, J. (1983), Instrumental problems in small gas turbine engines, in '19th Joint Propulsion Conference', p. 1293.

Bonham, C., Thorpe, S., Erlund, M. \& Stevenson, R. (2013), 'Stagnation temperature measurement using thin-film platinum resistance sensors', Measurement Science and Technology 25(1), 015101.

Coleman, H. W. \& Steele, W. G. (1995), 'Engineering applications of experimental uncertainty analysis', The American Institute of Aeronautics and Astronautics Journal 33(10), 1888-1896.

Cumpsty, N. \& Horlock, J. (2006), 'Averaging nonuniform flow for a purpose', Journal of Turbomachinery 128(1), 120-129.

Franz, A. (1940), Pressure and temperature measurement in supercharger investigations, Technical report, NACA.

Glawe, G. E., Krause, L. N. \& Dudzinski, T. J. (1968), A Small Combination Sensing Probe for Measurement of Temperature, Pressure, and Flow Direction, Vol. 4816, National Aeronautics and Space Administration.

Kiel, G. (1935), Total-head meter with small sensitivity to yaw, Technical report, National Advisory Committee for Aeronautics.

King, W. (1943), 'Measurement of high temperatures in high-velocity gas streams', Transactions of the American Society of Mechanical Engineers 65(5), 421.

Krause, L. N., Glawe, G. E. \& Dudzinski, T. J. (1972), 'A probe for measuring temperature and pressure at the same points in a gas stream'.

Lepicovsky, J. (2008), 'Effects of a rotating aerodynamic probe on the flow field of a compressor rotor', NASA/CR-2008-215215, E-16503 .

Markowski, S. \& Moffatt, E. (1948), Instrumentation for development of aircraft powerplant components involving fluid flow, Technical report, SAE International.

Massini, M., Miller, R. J., Hodson, H. P. \& Collings, N. (2010), A novel technique for measuring stagnation quantities and gas composition in high temperature flows, in 'ASME Turbo Expo 2010: Power for Land, Sea, and Air', American Society of Mechanical Engineers, pp. 223-233.

Moffat, R. J. (1962), Gas temperature measurement, in 'Temperature: Its Measurement and Control in Science and Industry', Vol. 1, p. 553.

Mullikin, H. (1941), 'Gas-temperature measurement and the high-velocity thermocouple', Temperature: Its measurement and control in science and industry p. 775.

Ng, H. C.-H. \& Coull, J. D. (2017), 'Parasitic loss due to leading edge instrumentation on a low-pressure turbine blade', Journal of Turbomachinery 139(4), 041007.

Otero, R., Lowe, K. T., Ng, W., Ma, L. \& Kim, C. (2016), Non-intrusive measurement of gas turbine engine exhaust characteristics using acoustic measurements, in '32nd AIAA Aerodynamic Measurement Technology and Ground Testing Conference', p. 4160.

Saravanamuttoo, H. (1990), Recommended practices for measurement of gas path pressures and temperatures for performance assessment of aircraft turbine engines and components, Technical report, Defense Technical Information Centre.

Stanworth, C. (1962), Suction pyrometers, in 'Proceedings of the Symposium on Some Developments in Techniques for Temperature Measurement', Vol. 1.

Thorpe, S. J., Bonham, C. \& Erlund, M. N. (2016), 'Total temperature probe'. US Patent 9,243,963.

Wilson, A. J., Ireland, P. T., Stevenson, R. J., Thorpe, S. J. \& Martin, D. (2012), A robust radial traverse temperature probe for application to a gas turbine HP/IP stage, in 'ASME Turbo Expo 2012: Turbine Technical Conference and Exposition', American Society of Mechanical Engineers, pp. $63-71$. 